\title{
A Pleuroperitoneal Shunt for Interactive Pleural Effusions With Yellow Nail Syndrome
}

\author{
Hiromasa Arai ${ }^{\mathrm{a}, \mathrm{e}}$, Kenji Inui ${ }^{\mathrm{a}}$, Teppei Nishii ${ }^{\mathrm{a}}$, Yoshitaka Oyama ${ }^{\mathrm{b}}$, Saoko Takeshita ${ }^{\mathrm{b}}$, \\ Masaaki Mori ${ }^{\mathrm{b}}$, Hiroaki Goto ${ }^{\mathrm{c}}$, Nobuyuki Kikuchib, \\ Takeshi Kaneko a , Munetaka Masuda ${ }^{\mathrm{d}}$
}

\begin{abstract}
Yellow nail syndrome is a relatively rare disease that is characterized by yellow nail, lymphedema and pleural effusion. These symptoms occur to various degrees, but occasionally pleural effusions are interactive and can become life-threatening. We report the case of a 15-year-old girl with yellow nail syndrome with interactive bilateral pleural effusions who was treated with pleuroperitoneal shunting. Drainage from the pleural cavity to the peritoneal cavity improved oxygenation and permitted removal of bilateral chest tubes that had been inserted for long term relief. We discuss the benefits and difficulties of this procedure and define the characteristics of patients with yellow nail syndrome who were treated with pleuroperitoneal shunt for pleural effusions, based on a literature review. The procedure may be an alternative rescue strategy for patients with interactive pleural effusions.
\end{abstract}

Keywords: Pleural effusion; Pleuroperitoneal shunt; Yellow nail syndrome

\section{Introduction}

Control of pleural effusion is very important for quality of

Manuscript accepted for publication March 30, 2011

${ }^{\text {a} R e s p i r a t o r y ~ D i s e a s e ~ C e n t e r, ~ Y o k o h a m a ~ C i t y ~ U n i v e r s i t y ~ M e d i c a l ~}$ Center, Japan

${ }^{\mathrm{b}}$ Children's Medical Center, Yokohama City University Medical Center, Japan

${ }^{c}$ Department of Pediatrics, Yokohama City University School of Medicine, Japan

${ }^{\mathrm{d}}$ Department of Surgery, Yokohama City University School of Medicine, Japan

${ }^{\mathrm{e} C}$ Corresponding author: Hiromasa Arai, 4-57 Urafune-cho, Minami-ku, Yokohama, 232-0024, Japan. Email: hiromasa@jg7.so-net.ne.jp

doi: $10.4021 / \mathrm{jmc} 202 \mathrm{w}$ life, but some interactive pleural effusions may be refractory to treatment. Chest tube insertion is the standard approach for control of pleural effusion, but pleurodesis, pleural abrasion, pleurectomy and pleural decortications are also used. The Denver shunt ${ }^{\circledR}$ (Denver Biomaterials Inc., Denver, CO) is a pleuroperitoneal shunt for control of pleural effusion, especially in benign disease. Here, we report the case of a patient with a rare disease, yellow nail syndrome, with interactive effusions that were treated with a pleuroperitoneal shunt.

\section{Case Report}

The patient was a 15-year-old girl who had been diagnosed with congenital general lymphedema at 9 years old. She was admitted to the Department of Pediatrics, Yokohama City University Hospital as an emergency case with a complaint of severe dyspnea, which had begun 1 week earlier. Chest $\mathrm{X}$-ray revealed large bilateral pleural effusion with a normal heart and pulmonary vasculature. She had no respiratory symptoms and no history of cardiac, renal, or hepatic disease. Chest tube insertion was performed on the right side and about $1,500 \mathrm{ml}$ of pleural effusion was removed. Her complaint immediately resolved.

The patient had a history of rhinosinusitis, mental retardation, epilepsy and short-term syncope. She had respiratory problems including pleural effusion and lymphedema, and these two conditions were sufficient for diagnosis of yellow nail syndrome, even though her fingernails and toenails were not markedly yellow.

A diagnosis of pleural effusion secondary to yellow nail syndrome was also made (Fig. 1a, b). There is no standard therapy for yellow nail syndrome, but prednisolone $(1 \mathrm{mg} /$ $\mathrm{kg} / \mathrm{day}$ ) and oral diuretics were administered based on previous reports. Lymphedema gradually improved over her whole body, with body weight loss of about $20 \mathrm{~kg} /$ month and a reduced frequency of syncope. Bilateral pleural effusions were also adequately controlled and the chest tube was removed. She was discharged from hospital and given further treatment as an outpatient. Oral prednisolone was slowly tapered to $7.5 \mathrm{mg} /$ day. 

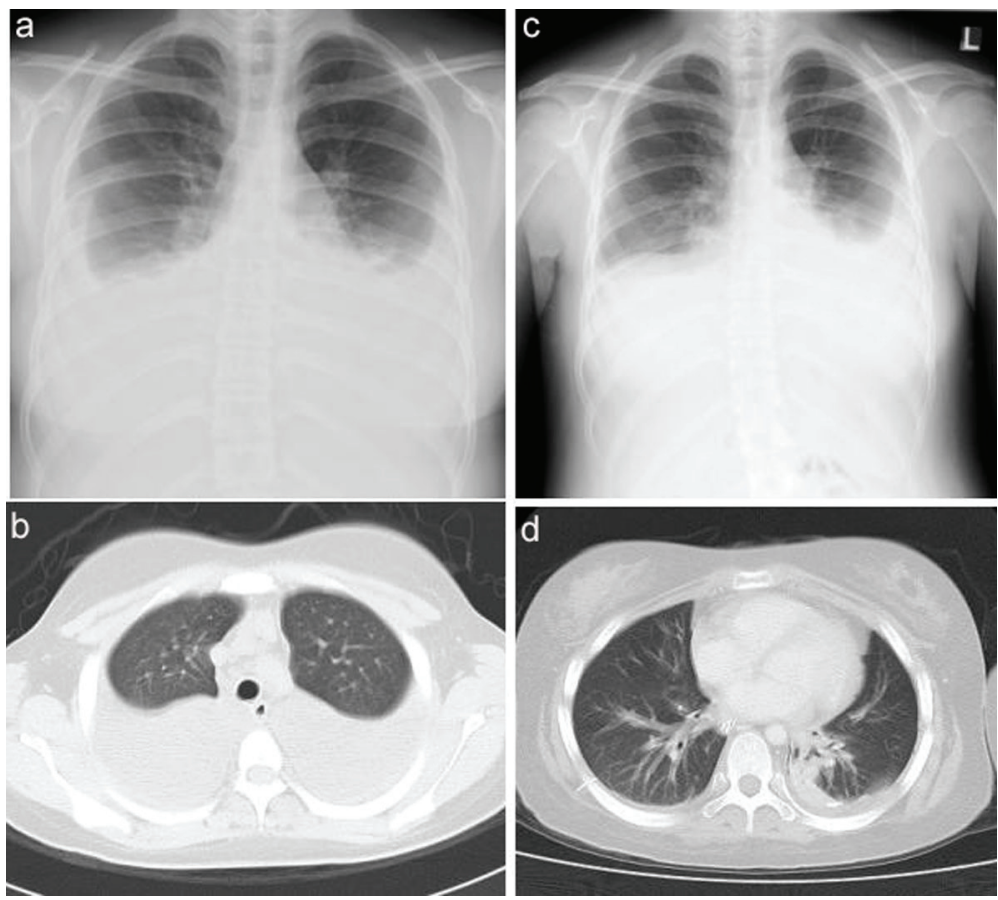

Figure 1. (a, b) Chest X-ray and chest CT on emergency admission, showing large bilateral pleural effusions. (c) Chest X-ray on emergency admission, showing left side dominant, bilateral pleural effusions. (d) Chest CT showing expanded lungs with a small atelectasis. Bilateral effusions were drained well by the two chest tubes.

Six months after discharge, the patient was admitted to the Children's Medical Center of our hospital as an emergency because of worsening of lymphedema of the whole body, a tendency for somnolence, and dyspnea. Chest X-ray showed recurrence of left side dominant, bilateral pleural effusions (Fig. 1c). The day after hospitalization, her consciousness worsened to E1V1M1 on the Glasgow Coma Scale (GCS). Routine chest X-ray showed increased bilateral pleural effusions and she developed respiratory failure. Intubation and respiratory management were performed in the intensive care unit (ICU), with chest tube insertion on both sides. Prednisolone $(1 \mathrm{mg} / \mathrm{kg} /$ day $)$ and diuretics were given by intravenous injection. Lymphedema gradually improved and her consciousness level improved to E3V3M4. The endotracheal tube was removed and the bilateral pleural effusions also decreased. The chest tubes were subsequently removed.

Two months later, the patient was re-admitted for the same condition, and underwent re-intubation, bilateral chest tube insertion, and steroid therapy in the ICU. Tracheotomy was also performed because of long-term respirator management for about one month. Her breathing gradually stabilized with only an oxygen supply, and without support of a respirator. She was discharged from the ICU and moved to a pediatric ward. However, the bilateral chest tubes could not be removed due to continuous drainage of large quantities of pleural effusion. She was then referred to our department
(Respiratory Disease Center) for control of the bilateral effusions.

Verbal communication was difficult because of her mental retardation and the tracheotomy. She required assistance in all aspects of daily life (performance status 4 ) because of cerebral infarction that had occurred in the ICU. She was put on a drip through a central vein catheter via the left internal jugular vein and took nourishment through enteral nutrition with a feeding tube. However, her consciousness was very clear, at E4VTM6 on the GCS. Bilateral pleural effusions were drained well by the chest tubes. Chest computed tomography (CT) showed expanded lungs with a small atelectasis and normal mediastinal structures (Fig. 1d).

The total volume of pleural effusion was about $300 \mathrm{ml} /$ day. The pleural effusion was clear and yellow, and the cell count was 2500 cells per $\mathrm{mm}^{3}$. Laboratory tests showed a relative density of 1.015 , a protein concentration of $1.6 \mathrm{~g} /$ $\mathrm{dl}$, a glucose concentration of $111 \mathrm{mg} / \mathrm{dl}$, and lactate dehydrogenase $(\mathrm{LDH})$ of $330 \mathrm{IU} / \mathrm{L}$. These results indicated transudative pleural effusion. Further tests showed levels of adenosine deaminase (ADA) of $13.6 \mathrm{IU} / \mathrm{L}$, hyaluronic acid of $24420 \mathrm{ng} / \mathrm{ml}$, carcinoembryonic antigen (CEA) of $3.0 \mathrm{ng} /$ $\mathrm{ml}, \alpha$-fetoprotein (AFP) of $1 \mathrm{ng} / \mathrm{ml}$, and amylase of $27 \mathrm{IU} / \mathrm{L}$. Routine bacterial smears and cultures and acid fast smears and cultures were negative. Cytological examination of the fluid was negative for tumor cells. A full blood examination showed slight pancytopenia (leukocytopenia of $2350 / \mathrm{mm}^{3}$, 


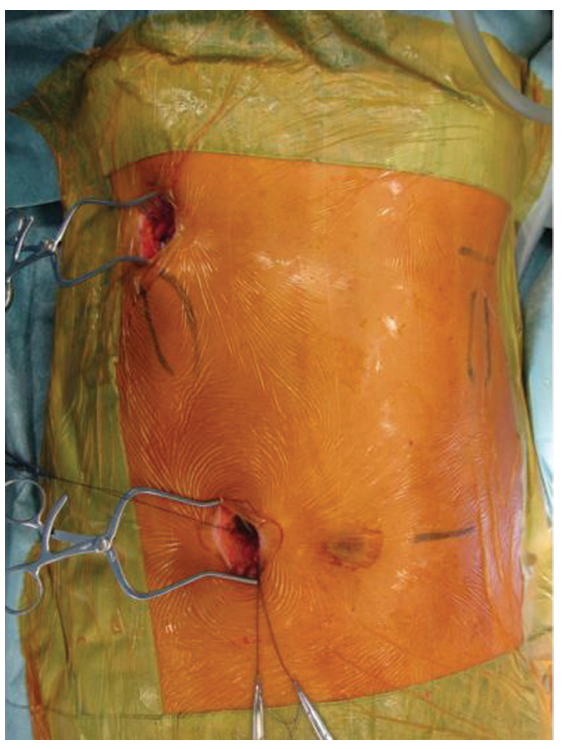

Figure 2. Skin incision on the inframammary line on the midclavicle line and a pararectal transverse skin incision at the umbilicus level on the abdomen.

hemoglobin of $9.0 \mathrm{~g} / \mathrm{dl}$ and a platelet count of $13.7 \times 10^{4} /$ $\mathrm{mm}^{3}$ ). Hypoproteinemia and hypoalbuminemia were also present, with total protein and serum albumin levels of $4.1 \mathrm{~g} /$ $\mathrm{dl}$ and $2.7 \mathrm{~g} / \mathrm{dl}$, respectively. The results of blood gas analysis were $\mathrm{pCO}_{2} 40.6 \mathrm{mmHg}$ and $\mathrm{pO}_{2} 78.9 \mathrm{mmHg}\left(\mathrm{O}_{2} 1 \mathrm{~L} / \mathrm{min}\right)$.

There are several techniques to control pleural effusion, including chemical pleurodesis, pleural abrasion, pleurectomy, and pleural decortications. However, these are difficult to perform and may not be successful without cooperation of the patient. Therefore, we selected bilateral plueroperitoneal shunting using the Denver shunt ${ }^{\circledR}$ under general anesthesia.

With the patient in the supine position on the table under general anesthesia, a skin incision of about $5 \mathrm{~cm}$ was made on the inframammary line on the midclavicle line of the right side. Subcutaneous tissue was dissected with a finger to make a pocket for placing the pumping chamber on the chest cage. With blunt hemostat dissection, the pleural cavity was entered in the fifth intercostal space. A pararectal transverse skin incision of about $5 \mathrm{~cm}$ was also made at the umbilicus level in the abdomen. The pararectal sheath was incised. A pursestring suture was placed in the posterior pararectal sheath and peritoneum. A subcutaneous tunnel was created between the inframammary skin incision and the pararectal incision in the abdomen with blunt hemostat dissection. The receiving site of the shunt (short tube) was introduced into the pleural cavity from the fifth intercostal space. The delivery end of the shunt (long tube) was delivered through the subcutaneous tunnel and inserted into the peritoneal cavity. The pursestring suture was tightened. The pumping chamber was installed into the subcutaneous pocket, sutured in place using non-absorbable monofilament, and fixed well on the chest cage. Compression of the pumping chamber caused fluid flow from the pleural cavity to the peritoneal cavity. Finally, the suitability of the location of the shunt system was confirmed by portable X-ray illumination, and wounds were closed (Fig. 2). The procedure on the left side was performed using the same method. After the operation, bilateral chest tubes were clamped and the patient was asked to compress the pumping chamber, which rested on the chest cage, but she was unable to do this. Therefore, the doctor or nurse in attendance performed this task. Daily X-rays indicated that by slowly increasing the frequency of pumping, chamber pumping for approximately 5 min two times per day on each
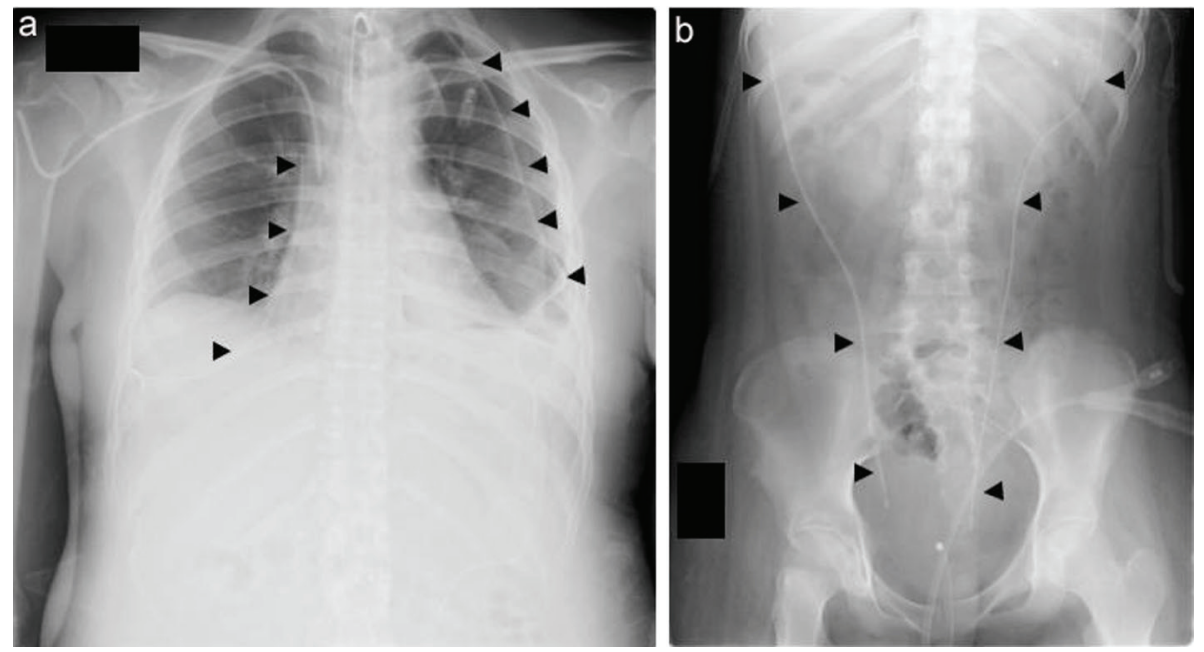

Figure 3. Postoperative X-ray findings. (a) Chest X-ray showing the bilateral receiving sites of Denver shunts in both pleural cavities (arrowheads). Pleural effusions were unremarkable. (b) Abdominal X-ray showing the delivery ends of the shunts (arrowheads). 
side, a frequency which theoretically results in fluid transfer of up to $1000 \mathrm{ml} / 24 \mathrm{~h}$, was required for adequate control of the bilateral pleural effusion (Fig. 3a, b). When oxygen saturation deteriorated, oxygenation was improved by additional manual compression of the pump chamber. All chest tubes were removed on the 22nd postoperative day. The patient's family provided informed consent regarding publication of the features of this case.

\section{Discussion}

Yellow nail syndrome is characterized by yellow (discolored) nail, lymphedema and chronic respiratory manifestations, as first described in 1964 by Samman and White [1]. There have been approximately 150 reported cases of this syndrome up to 2009 [2]. It is now accepted that diagnosis can be made based on the presence of just two of the following symptoms: slow-growing nails $(<0.5 \mathrm{~mm} /$ week $)$ or abnormal nails (pale yellow/green nails, onycholysis), respiratory problems (including pleural effusion), and lymphedema [2, 3]. The clinical features vary among individuals: Maldonado et al. [4] suggested that all three criteria may not be simultaneously present and Beer et al. [5] found that 9 of 20 patients diagnosed with yellow nail syndrome had lymphedema and pleural effusion without nail abnormalities. The gap between onset of symptoms may also vary, and Ce'ligny et al. [6] described a patient with repeated bilateral effusions who developed yellow fingernails and toenails and slight lymphedema of both lower limbs 14 years later.

Multiple clinical associations with yellow nail syndrome have been reported, including connective tissue disease, malignancy, immunodeficiency status, drugs, and several endocrine disorders including diabetes mellitus and thyroid dysfunction [4]. Anti-rheumatic therapy with $d$-penicillamine [7] and bucillamine [8] may induce yellow nail syndrome, and the related respiratory manifestations include bronchiectasis, rhinosinusitis, chronic cough, recurrent lung infections and pleural effusion [1].

Pleural effusion in yellow nail syndrome has been reported to occur at rates of 36\% [9], 40\% [2], 46\% [4] and $63 \%$ [3] and is sometimes chylous [10] or is chylous in $30 \%$ of all cases with pleural effusion [2]. The age at onset of symptoms is typically between the 4 th and 6 th decades [2, 4], but Nordkild et al. [11] described cases from a newborn with lymphedema to patients of 65 years old.

Hypoplastic lymphatics [1], impaired lymphatic drainage [11], and dilated lymphatic vessels on electron microscopy [6] have been hypothesized as the etiology of yellow nail syndrome, but the pathophysiology remains unclear and there is no standard therapy. Antibiotic therapy for respiratory infections, oral vitamin E therapy for yellow nail, elastic bandages or diuretic treatment for lymphedema, and general steroid therapy $[4,5,11]$ have been attempted, but the cura-

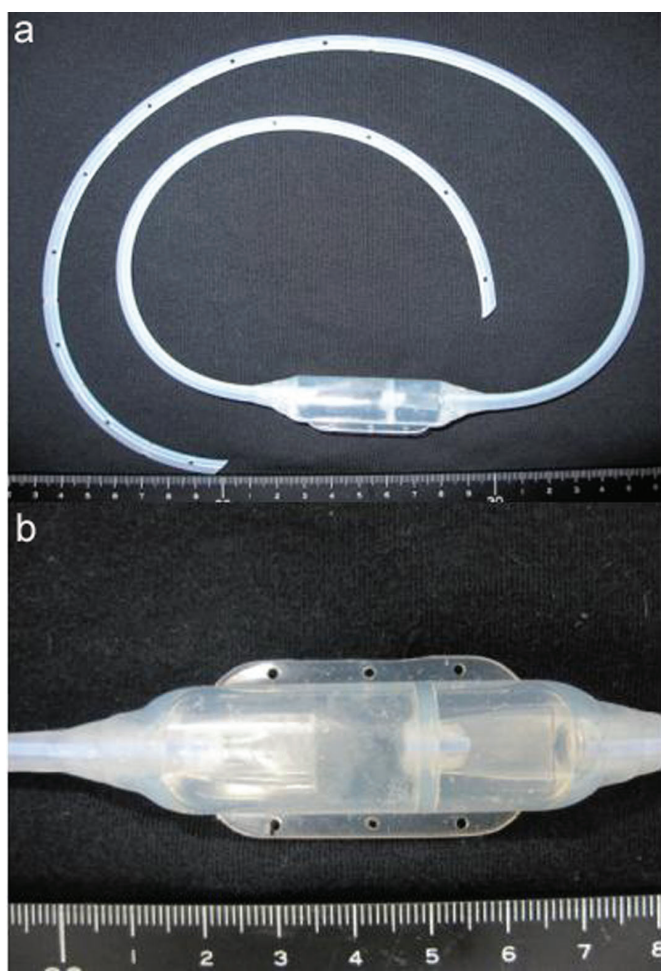

Figure 4. Denver shunt ${ }^{\circledR}$. (a) The short tube (receiving site) is installed in the pleural cavity and the long tube (delivery end) is installed in the peritoneal cavity. (b) The pump chamber containing two one-way valves is installed on the chest cage.

tive efficacy of these approaches is uncertain.

The natural history of yellow nail syndrome can also be extremely unusual. Ce'ligny et al. [6] described a case in which bilateral pleural effusions disappeared spontaneously and yellow discoloration progressively decreased after left parietal pleurectomy for recurrent bilateral pleural effusions. Maldonado et al. [4] found that yellow nails improved, often after specific therapy, in about half of 41 consecutive patients.

Pleural effusion causing few or no symptoms may not require thoracentesis or may need treatment only occasionally. However, a large amount or rapid accumulation of pleural effusion can cause respiratory failure and must be treated. Approaches include chemical pleurodesis by infusing a sclerosing agent into the pleural cavity to obliterate the pleural space, pleural abrasion, pleurectomy and pleural decortication. However, these techniques are not always successful and may cause difficulty for future surgery in a patient who subsequently develops other lung diseases that require an operation.

In pleuroperitoneal shunting, the pleural effusion is guided from the pleural cavity to the abdominal space, where (in principle) it can be absorbed by the large peritoneum surface. The Denver pleuroperitoneal shunt system was de- 
Table 1. Characteristics of Reported Cases of Yellow Nail Syndrome With Pleural Effusion Treated Using a Pleuroperitoneal Shunt

\begin{tabular}{|c|c|c|c|c|c|c|c|}
\hline Case & Year & First author & $\begin{array}{l}\text { Age } \\
\text { (years) }\end{array}$ & Sex & $\begin{array}{l}\text { Effusion } \\
\text { (site/shunt site) }\end{array}$ & Complications & Outcome \\
\hline 1 & 1990 & Brofman [12] & 65 & $\mathrm{~F}$ & $1 t / 1 t$ & $\begin{array}{l}\text { leg edema, yellow } \\
\text { nail }\end{array}$ & $\begin{array}{l}\text { replaced due to } \\
\text { obstruction }\end{array}$ \\
\hline 2 & 2004 & Moorjani [3] & 75 & $\mathrm{~F}$ & bil/bil & $\begin{array}{l}\text { leg edema, yellow } \\
\text { nail }\end{array}$ & $\begin{array}{l}\text { replaced due to } \\
\text { infection, then patent } \\
\text { for } 8 \text { years }\end{array}$ \\
\hline 3 & 2005 & Tanaka [10] & 70 & M & $\mathrm{bil}^{\mathrm{b}} / \mathrm{rt}$ & $\begin{array}{l}\text { general edema, } \\
\text { yellow nail }\end{array}$ & $\begin{array}{l}\text { replaced with a } \\
\text { pleurovenous shunt }\end{array}$ \\
\hline $4^{a}$ & 2007 & Katsura [14] & 56 & M & $\mathrm{bil} / \mathrm{rt}$ & BOOP, yellow nail & effective \\
\hline 5 & 2010 & Present case & 15 & $\mathrm{~F}$ & bil/bil & $\begin{array}{l}\text { general edema, } \\
\text { rhinosinusitis mental } \\
\text { retardation, epilepsy, } \\
\text { syncope }\end{array}$ & effective \\
\hline
\end{tabular}

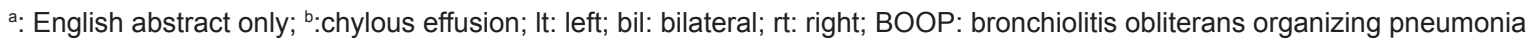

veloped in 1984 (Fig. 4a, b), and consists of a fenestrated pleural catheter, a flexible pump chamber containing two miter valves, and a fenestrated peritoneal catheter. The entire shunt is constructed from medical-grade silicone rubber and an integral barium sulfate stripe in the wall of both catheters permits visualization by X-ray to confirm proper positioning during surgery. The pump chamber has two one-way valves, and pleural effusion begins to flow automatically when the intrathoracic pressure exceeds the intraabdominal pressure by more than $3 \mathrm{cmH}_{2} \mathrm{O}$. Each complete pump of the pump chamber (manual compression) will transfer approximately $2 \mathrm{ml}$ of fluid. Steady pumping will transfer approximately $250 \mathrm{ml}$ of fluid in 5 minutes.

In our case, we selected bilateral plueroperitoneal shunting using the Denver shunt ${ }^{\circledR}$. A pleurovenous shunt was not chosen because the patient had undergone central vein catheter insertion many times via the bilateral subclavian veins, and these veins were obstructed or narrowed by thrombus.

Brofman et al. reported the first use of a pleuroperitoneal shunt for yellow nail syndrome in 1990 [12]. A PubMed search revealed only 4 reports of use of a pleuroperitoneal shunt for this syndrome [10, 12-14]. Including our case, the clinical features of the 5 reported cases are summarized in Table 1 . The patients ( 2 men and 3 women) ranged in age from 15 to 75 years old, with a mean age of $40 \pm 35.4$ years old. All patients except our case had yellow, discolored nails, and 4 had bilateral pleural effusions, including one with chylous effusion. Bilateral chest tube insertion was used in only 2 cases, including our case. Shunt problems such as obstruction, infection and replacement with a pleurovenous shunt due to marked abdominal distention occurred in 3 cases.

Problems and complications with this procedure include infection, shunt occlusion, and shunt failure. Shunt occlusion is a particular problem, and judgment of the patency of the tube may be difficult. The manufacturer recommends testing the shunt function by a non-invasive technique, by compressing the pump chamber firmly with the fingers to determine whether there is resistance and observing whether the pump chamber fails to refill. In our case, we confirmed fluid flow using color Doppler ultrasonography through the body surface. This inspection is noninvasive, simple, and very useful. Shuntography with contrast medium introduced into the pump chamber using a fine needle injection via the body surface may also be used to confirm the patency of the shunt tube. The tube should be removed in a case of infection and if pleuroperitoneal drainage of effusion exceeds the capacity of absorption of the peritoneum surface or drainage is not effective. Clinically, this results in marked abdominal distention and replacement with a pleurovenous shunt is necessary.

The patency and durability of the components, training of patients and helpers, and the need for manual compression until the primary disease is controlled adequately may also cause problems. Moorjani et al. [13] reported that the shunt remains patent for 8 years, but there is no major collective experience of this procedure. Long-term follow up and accumulation of patients will allow appropriate countermeasures to be developed to address the complications.

Use of a pleuroperitoneal shunt for malignant disease is still controversial, but we believe that this procedure is extremely useful for non-malignant diseases such as idiopathic/interactive chylothorax, cirrhosis of the liver, idiopathic portal hypertension, and interactive pleural effusion (including chylous effusion) in yellow nail syndrome. It may also be possible to perform the procedure using the Denver shunt kit under local anesthesia.

The procedure is relatively simple and easy, and can be performed by many thoracic surgeons. If the primary disease improves, the shunt can be removed immediately. Therefore, the pleuroperitoneal Denver shunt may be a useful alternative rescue therapy for patients with interactive pleural effusions associated with non-malignant disease. 


\section{Conflict of Interest}

We declare that we have no conflict of interest.

\section{References}

1. Samman PD, White WF. The "Yellow Nail" Syndrome. Br J Dermatol 1964;76:153-157.

2. Maldonado F, Ryu JH. Yellow nail syndrome. Curr Opin Pulm Med 2009;15(4):371-375.

3. Hershko A, Hirshberg B, Nahir M, Friedman G. Yellow nail syndrome. Postgrad Med J 1997;73(862):466-468.

4. Maldonado F, Tazelaar HD, Wang CW, Ryu JH. Yellow nail syndrome: analysis of 41 consecutive patients. Chest 2008;134(2):375-381.

5. Beer DJ, Pereira W, Jr., Snider GL. Pleural effusion associated with primary lymphedema: a perspective on the yellow nail syndrome. Am Rev Respir Dis 1978;117(3):595-599.

6. Solal-Celigny P, Cormier Y, Fournier M. The yellow nail syndrome. Light and electron microscopic aspects of the pleura. Arch Pathol Lab Med 1983;107(4):183-185.

7. Ilchyshyn A, Vickers CF. Yellow nail syndrome associated with penicillamine therapy. Acta Derm Venereol 1983;63(6):554-555.

8. Tanaka H, Natsuda M, Noguchi M, Tanaka C, Taniuchi $\mathrm{K}$. A case of yellow nail syndrome induced by bucilla- mine (in Japanese with English abstract). Rheumatology 2006;35:309-313.

9. Hiller E, Rosenow EC, 3rd, Olsen AM. Pulmonary manifestations of the yellow nail syndrome. Chest 1972;61(5):452-458.

10. Tanaka E, Matsumoto K, Shindo T, Taguchi Y. Implantation of a pleurovenous shunt for massive chylothorax in a patient with yellow nail syndrome. Thorax 2005;60(3):254-255.

11. Nordkild P, Kromann-Andersen H, Struve-Christensen E. Yellow nail syndrome--the triad of yellow nails, lymphedema and pleural effusions. A review of the literature and a case report. Acta Med Scand 1986;219(2):221227.

12. Brofman JD, Hall JB, Scott W, Little AG. Yellow nails, lymphedema and pleural effusion. Treatment of chronic pleural effusion with pleuroperitoneal shunting. Chest 1990;97(3):743-745.

13. Moorjani N, Winter RJ, Yigsaw YA, Maiwand MO. Pleural effusion in yellow nail syndrome: treatment with bilateral pleuro-peritoneal shunts. Respiration 2004;71(3):298.

14. Katsura H, Nakagawa K, Iwasaki T, Tamura M, Nakane S. Throracoscopic assisted pleuro-peritoneal shunt placement procedure for patient having refractory pleural effusion: a case report of "yellow-nail syndrome" (in Japanese with English abstract). Nihon kokyukigeka gakkai zasshi 2007;21:565-570. 